\title{
Non-linear Poisson-Boltzmann Theory for Swollen Clays
}

\author{
R. J. F. Leote de Carvalho (1), E. Trizac (1)* and J.-P. Hansen (2) \\ (1) Laboratoire de Physique, Ecole Normale Supérieure de Lyon (URA CNRS 1325) \\ 46 Allée d'Italie, 69364 Lyon Cedex 07, France \\ (2) Department of Chemistry, University of Cambridge \\ Lensfield Road, Cambridge CB2 1EW, UK
}

(21 April 1998)

\begin{abstract}
PACS. 02.60.Nm - Integral and integrodifferential equations
PACS. 82.70.Gg - Gels and sols

PACS. 68.10.-m - Fluid surfaces and fluid-fluid interfaces
\end{abstract}

\begin{abstract}
The non-linear Poisson-Boltzmann (PB) equation for a circular, uniformly charged platelet, confined together with co- and counter-ions to a cylindrical cell, is solved semi-analytically by transforming it into an integral equation and solving the latter iteratively. This method proves efficient and robust, and can be readily generalized to other problems based on cell models, treated within non-linear Poisson-like theory. The solution to the PB equation is computed over a wide range of physical conditions, and the resulting osmotic equation of state is shown to be in fair agreement with recent experimental data for Laponite clay suspensions, in the concentrated gel phase.
\end{abstract}

\footnotetext{
${ }^{*}$ Present address: FOM Institute for Atomic and Molecular Physics, Kruislaan 407, 1098 SJ Amsterdam, The Netherlands
} 
Upon the addition of water, initially dry clays swell into stacks of parallel charged platelets separated by increasingly thick layers of water containing microscopic co- and counter-ions. This simple picture becomes less realistic as the concentration by weight of clay decreases and the initial smectic orientational order is gradually lost in favor of a disordered gel structure. The development of this gel phase is responsible for characteristic rheological properties of the clay dispersion, which are very sensitive to salt concentration and of considerable technological importance, in particular for the oil drilling industry. Recent experimental work [1-3] focussed on correlating the rheological behavior with the mesoscopic structure of the well-characterized synthetic clay Laponite, made of quasimonodisperse disc-like platelets of thickness $\approx 1 \mathrm{~nm}$, diameter $\sigma \approx 30 \mathrm{~nm}$, and carrying a negative surface charge $-Z e \approx-10^{3} e$ (where $e$ is the proton charge). The complex interplay of excluded volume and electrostatic interactions between such highly anisotropic particles makes a theoretical description of clay gels extremely difficult [1]. However, the early stages of swelling are described reasonably well by a cell model where each platelet, assumed to be an infinite uniformly charged plane, is confined with its monovalent co- and counter-ions to a slab, and the distribution of the latter is treated within Poisson-Boltzmann (PB) theory [5]. As swelling proceeds the spacing between platelets becomes a sizeable fraction of their lateral dimension. Consequently, edge (finite size) effects become important and the problem ceases to be one-dimensional. In the extreme case of an isolated platelet immersed in an electrolyte solution (infinite dilution limit), the PB problem can be solved numerically [6.7]. So far, these results provide the only available data for disc-like clays and no solution could be obtained for finite concentrations of platelets. In this Letter we report results for a cylindrical cell model, which accounts for both the finite size of the platelets and their finite concentration, and allows a semi-analytical treatment within non-linear PB theory. The linearized version of PB theory (LPB) for a circular platelet confined with its co- and counter-ions to the same cell has been recently considered in detail [8]; however, LPB theory is not suited for large surface potentials, typically for $Z>10^{2}$, which is an order of magnitude down from physical Laponite charges, where linearization is no longer justified. 
Motivated by the results of [3], we introduce the Wigner-Seitz cell around a clay platelet, and we consider that the average shape of this confining cell is cylindrical in the gel phase. If $n$ is the number concentration of platelets, the volume of the cylindrical cell is $v=1 / n=$ $2 \pi R^{2} h$ where $R$ is the radius and $2 h$ the height, which is the spacing between platelets in the stack (typically of the order of $10 \mathrm{~nm}$; however $h$ is not a priori prescribed in our model). The platelets are assumed to be infinitely thin, of radius $r_{0}$, and carrying a uniform surface charge density $\sigma=-Z e / \pi r_{0}^{2}$. Let $\rho^{+}(\mathbf{r})$ and $\rho^{-}(\mathbf{r})$ denote the counter-ion and co-ion density profiles inside the cell. The electrostatic potential $\varphi(\mathbf{r})$ satisfies Poisson's equation:

$$
\nabla^{2} \varphi(\mathbf{r})=-\frac{4 \pi}{\epsilon}\left(q_{P}(\mathbf{r})+e\left[\rho^{+}(\mathbf{r})-\rho^{-}(\mathbf{r})\right]\right)
$$

where $q_{P}(\mathbf{r})=\sigma \delta(z) \theta\left(r_{0}-r\right)$ is the charge density of the platelet in cylindrical coordinates, and $\epsilon$ is the macroscopic dielectric constant of water (primitive model). Within mean-field, or PB theory, the density profiles are approximated by the Boltzmann factors:

$$
\rho^{ \pm}(\mathbf{r})=\rho_{0}^{ \pm} \exp [\mp \beta e \varphi(\mathbf{r})]
$$

where $\beta \equiv 1 / k_{B} T$ is the inverse temperature. The pre-factors $\rho_{0}^{ \pm}$have individually no physical significance, while the product $\rho_{0}^{+} \rho_{0}^{-}$depends on the conditions under consideration. If the salt concentration $n_{S}$ is assumed to be fixed in the dispersion, then the concentration of co- and counter-ions in the cell are imposed: $n_{-}=N_{-} / v=n_{S}$ and $n_{+}=N_{+} / v=n_{S}+Z / v$, and the $\rho_{0}^{ \pm}$are determined by the normalization constraints (canonical description):

$$
\frac{1}{v} \int_{v} \rho^{ \pm}(\mathbf{r}) d \mathbf{r}=n_{ \pm}
$$

On the other hand, if the dispersion is in equilibrium with an ionic solution of concentration $n_{S}^{\prime}$, which acts as a reservoir, then the sum of the electrochemical potentials of co- and counter-ions in the cell must equal the same quantity for the salt in the reservoir. Since, for consistency, the ions in the cell are assumed to behave as an ideal solution, chemical equilibrium implies that [5] (semi-grand-canonical description):

$$
\rho_{0}^{+} \rho_{0}^{-}=\left(n_{S}^{\prime}\right)^{2}
$$


By substituting (21) into (11), and subtracting $\kappa^{2} \varphi(\mathbf{r})$ (where $\kappa$ is an arbitrary inverse length), we arrive at the closed non-linear partial differential equation for the potential $\varphi(\mathbf{r}):$

$$
\left(\nabla^{2}-\kappa^{2}\right) \varphi(\mathbf{r})=-\frac{4 \pi}{\epsilon}\left(q_{P}(\mathbf{r})+e \rho_{0}^{+} \mathrm{e}^{-\beta e \varphi(\mathbf{r})}-e \rho_{0}^{-} \mathrm{e}^{\beta e \varphi(\mathbf{r})}+\frac{\epsilon \kappa^{2}}{4 \pi} \varphi(\mathbf{r})\right)
$$

Bearing upon the physical significance of the cell model, it is assumed that the normal component of the electric field vanishes everywhere on the surface $\Sigma$ bounding the cylinder cell. Therefore, the boundary condition for the potential $\varphi(\mathbf{r})$ is:

$$
[\mathbf{n}(\mathbf{r}) \cdot \nabla]_{\mathbf{r} \in \Sigma} \varphi(\mathbf{r})=0
$$

where $\mathbf{n}(\mathbf{r})$ denotes the normal to the surface $\Sigma$ at point $\mathbf{r}$. The solution of (5) satisfies:

$$
\varphi(\mathbf{r})=-\frac{4 \pi}{\epsilon} \int G^{\kappa}\left(\mathbf{r}, \mathbf{r}^{\prime}\right)\left(q_{P}\left(\mathbf{r}^{\prime}\right)+e \rho_{0}^{+} \mathrm{e}^{-\beta e \varphi\left(\mathbf{r}^{\prime}\right)}-e \rho_{0}^{-} \mathrm{e}^{\beta e \varphi\left(\mathbf{r}^{\prime}\right)}+\frac{\epsilon \kappa^{2}}{4 \pi} \varphi\left(\mathbf{r}^{\prime}\right)\right) d \mathbf{r}^{\prime}
$$

where $G^{\kappa}\left(\mathbf{r}, \mathbf{r}^{\prime}\right)$ is the Green's function which solves the linear problem:

$$
\left(\nabla^{2}-\kappa^{2}\right) G^{\kappa}\left(\mathbf{r}, \mathbf{r}^{\prime}\right)=\delta\left(\mathbf{r}-\mathbf{r}^{\prime}\right)
$$

subject to the same boundary condition (6). The reason for replacing the bare Laplace operator by the screened Laplace operator is that for $\kappa=0$ the boundary condition (6) cannot be satisfied by the solution of eq. (8), as becomes clear by integrating both sides of eq. (8) (with $\kappa=0$ ) over the cell volume $v$. The Neuman-like boundary condition allows the Green's function to be expanded, in cylindrical coordinates, in the form of a Bessel-Dini series, along the lines of the procedure for obtaining the solutions to the LPB problems in [8]. We find:

$$
G^{\kappa}\left(\mathbf{r}, \mathbf{r}^{\prime}\right)=\sum_{n \geq 1} \mathcal{C}_{n}^{ \pm}\left(\phi, \mathbf{r}^{\prime}\right) \cosh \left[\frac{h \mp z}{\Lambda_{n}}\right] J_{0}\left(y_{n} \frac{\rho}{R}\right)
$$

where $\mathbf{r}=(\rho, \phi, z)$, the + and - signs correspond to the situations $z>z^{\prime}$ and $z<z^{\prime}$ respectively, $y_{n}$ is the $\mathrm{n}^{\text {th }}$ root of $J_{1}(y)=0, J_{0}$ and $J_{1}$ are the zeroth and first order Bessel functions, $\Lambda_{n}^{-2}=\left(y_{n}^{2}+\kappa^{2} R^{2}\right) / R^{2}$, and: 


$$
\mathcal{C}_{n}^{ \pm}\left(\phi, \mathbf{r}^{\prime}\right)=-\frac{2 \Lambda_{n} J_{0}\left(y_{n} \rho^{\prime} / R\right) \delta\left(\phi-\phi^{\prime}\right)}{R^{2} J_{0}^{2}\left(y_{n}\right) \sinh \left[2 h / \Lambda_{n}\right]} \cosh \left[\frac{h \pm z^{\prime}}{\Lambda_{n}}\right]
$$

The kernel $G^{\kappa}$ being explicitly known, the non-linear integral equation (7) may be solved for $\varphi(\mathbf{r})$ by an iterative Picard procedure starting from an initial guess of $\varphi(\mathbf{r})$ (in most cases we have chosen either $\varphi(\mathbf{r}) \equiv 0$ or the solution of LPB theory [8]). The arbitrary inverse length $\kappa$ has generally been chosen in the range $\left[\kappa_{D} / 10,10 \kappa_{D}\right], \kappa_{D}$ being the inverse Debye length in the reservoir. Cylindrical symmetry implies $\varphi(\mathbf{r})=\varphi(\rho, z)$, which is calculated on a two-dimensional $n_{\rho} \times n_{z}$ grid spanning half the cylinder, with $n_{\rho}=200$ grid points to cover the interval $[0, R]$ (this must be sufficiently large to allow for a proper representation of the Bessel functions), and $n_{z}=40$ points for the interval $[0, h] .50$ terms were retained in the Bessel-Dini series (9), and at each step of the iteration typically $10 \%$ to $5 \%$ of the new estimate of $\varphi(\mathbf{r})$ were mixed with $90 \%$ to $95 \%$ of the previous estimate to ensure proper convergence; the latter was generally achieved in about 50 to 200 iterations to ensure a relative accuracy of at least $10^{-5}$. Examples of the resulting density profiles are shown in Fig. 1 and compared with the LPB predictions; under realistic conditions the deviations of LPB from PB theory are indeed considerable. Provided the iterative procedure converges, the method yields a solution independent of $\kappa$, i.e. no limit $\kappa \rightarrow 0$ is required at the end. We have successfully implemented two independent checks for the accuracy of the computed potential. First, eq. (7) must be satisfied for any values of $\kappa$. Secondly, since the multipoles of the total charge distribution inside the cell can be transformed from volume integrals (i.e. their definition) into surface integrals (making use of Poisson's equation), these two formulae must yield the same result provided the potential $\varphi(\mathbf{r})$ is a solution of the PB problem.

Once the potential $\varphi(\mathbf{r})$ is known for different surface charge densities $\sigma$, the Helmholtz free energy $F$ can be calculated by a charging process, e.g. at constant Debye length [8]. In the semi-grand-canonical calculations, the number of co- and counter-ions, $N_{-}$and $N_{+}$, are computed from the density profiles (for a given salt concentration $n_{S}^{\prime}$ in the reservoir), and the grand potential $\Omega$ is estimated from:

$$
\Omega=F-2 N_{-} k_{B} T \ln \left[n_{S}^{\prime} \lambda^{3}\right]
$$


where $\lambda$ is the de Broglie thermal wavelength.

The total quadrupole moment $Q$ of the charge distribution in the cell (first non-vanishing multipole), the osmotic pressure $\Pi$ and the disjoining pressure $\Pi_{d}$, can also be calculated from the density profiles as described in ref. [8]. For a given clay concentration $n$, and hence cell volume $v$, all these quantities depend on the aspect ratio $h / R$ (or equivalently $h / r_{0}$ ) of the cell. The equilibrium stacking is determined by minimizing either the free energy or the grand potential with respect to $h / r_{0}$. This is illustrated in Fig. 2. As expected, $h$ is found to be of the order of $10 \mathrm{~nm}$. The minima from the PB calculations turn out to be consistently flatter than the LPB results, pointing to the possibility of large fluctuations of the stacking configurations. The optimum aspect ratios $h / r_{0}$ predicted by PB and LPB are practically identical. This ratio varies with $n$, but is very insensitive to salt concentration. As in the LPB case, $Q$ is found to vanish at the minimum of the relevant thermodynamic potential ( $F$ in the canonical case or $\Omega$ in a semi-grand-canonical description, the latter being suited for a comparison with the experiments of [2]), while $\Pi$ and $\Pi_{d}$ are equal at that point. This property is exact and may serve as a check for the consistency of the numerical calculations, while the vanishing of $Q$ cannot be proven a priori within the $\mathrm{PB}$ framework, but may be regarded as an indication of the validity of our cell model for the description of dispersions of clay platelets [8].

Our PB results for the osmotic equation of state, $\Pi$ versus clay concentration, are compared in Fig. 3 to the experimental data of Mourchid et al. 22 for three reservoir salt concentrations $n_{S}^{\prime}$. The agreement is reasonable at the higher concentrations (above $C \approx$ $4 \%(w / w)$ in units of $\%$ of weight of Laponite per weight of solvent (water)) corresponding to the gel phase, except for the lowest salinity investigated $\left(n_{S}^{\prime}=10^{-4} \mathrm{M}\right.$ with $1 \mathrm{M}=1 \mathrm{~mol}$ $\mathrm{dm}^{-3}$ ) for which the convergence criterion is more difficult to meet. At this low salinity the PB predictions overestimate the osmotic pressure considerably, compared to the experiments. This disagreement may be linked to an excessive depletion of salt (Donnan effect) predicted by PB theory (see Table 1). Too strong a depletion leads to insufficient screening, and hence to an overestimation of the osmotic pressure. 
The cell model should not apply at low clay concentrations where the parallel stacking of platelets is completely lost, giving rise to the orientationally disordered sol phase. Since there is no long-ranged orientational order in the gel phase [2, [3] our cell model tends to overestimate the effect of lamellar order. However, the analytical results obtained in [8] within LPB indicate that given a clay concentration, and at least at this level of approximation, the precise shape of the confining cell does not affect significantly mesoscopic or macroscopic quantities such as the osmotic pressure. Finally, note that LPB theory leads to negative osmotic pressures under most conditions represented in the figure.

In order to assess the importance of edge effects we have also considered the case of infinite planar platelets having the same charge and mass density than the finite Laponite discs. The corresponding one-dimensional PB problem can be solved numerically along the lines in ref. [5]. For a given concentration by weight in Laponite the spacing $2 h_{\infty}$ between the infinite platelets differs from the optimum (lowest free energy) spacing between the finite discs (which is practically independent of $n_{S}^{\prime}$ ). A comparison is made in Fig. 3. It is clear that as $n_{S}^{\prime}$ increases edge effects become more important and the simple model of a stack of charged layers cannot describe the structure of the Laponite suspension. The two curves for the osmotic pressure as obtained from this simple model at the higher concentrations $n_{S}^{\prime}$ are completely off scale. In view of the poor performance of PB theory for finite platelets at $n_{S}^{\prime}=10^{-4} \mathrm{M}$, the reasonable agreement of the osmotic pressure calculated for infinite platelets with experimental data must be considered as fortuitous.

In summary, the present PB calculations confirm the qualitative features of earlier LPB results but while the latter predictions for the osmotic pressure are unphysical, the PB results are in reasonable agreement with experimental data for Laponite in the high concentration regime. The transformation of the initial Poisson equation into an integral equation problem, combined with the analytical calculation of the corresponding Green's function, proves efficient and can be readily generalized to other Poisson problems with non-linear source terms.

We thank Thierry Biben for many stimulating discussions. RJFLdC is presently carrying 
out work at the ENS de Lyon as part of a project financed by the European Commission through the Training and Mobility of Researchers (TMR) programme. ET acknowledges the hospitality of Professor Daan Frenkel (AMOLF, Amsterdam). 


\section{REFERENCES}

[1] Ramsay J.D.F. and Linder P., J. Chem. Soc. Faraday Trans., 89 (1993) 4207.

[2] Mourchid A., Delville A., Lambard J., LÉcolier E. and Levitz P., Langmuir, 11 (1995) 1942.

[3] Pignon F., Magnin A., Piau J.-M., Cabane B., Lindner P. and Diat O., Phys. Rev. E, 56 (1997) 3281.

[4] For an early attempt based on a very crude model see: DiJKstra M., Hansen J.-P. and Madden P., Phys. Rev. E, 55 (1997) 3044.

[5] Dubois M., Zemb T., Belloni L., Delville A., Levitz P. and Setton R., J. Chem. Phys., 96 (1992) 2278.

[6] Secor R. B. and Radke C. J., J. Colloid Interface Sci., 103 (1985) 237.

[7] Chang F. R. C. and Sposito G., J. Colloid Interface Sci., 163 (1994) 19.

[8] Trizac E. and Hansen J.-P., Phys. Rev. E, 56 (1997) 3137. 


\section{TABLES}

\begin{tabular}{c|cccc}
\hline \hline & \multicolumn{4}{|c}{ Values of $n_{S} / n_{S}^{\prime}$ at } \\
$C \%(w / w)$ & $n_{S}^{\prime}=10^{-4} \mathrm{M}$ & $n_{S}^{\prime}=10^{-3} \mathrm{M}$ & $n_{S}^{\prime}=5 \times 10^{-3} \mathrm{M}$ & $n_{S}^{\prime}=10^{-2} \mathrm{M}$ \\
\hline 3.293 & 0.025 & 0.238 & 0.654 & 0.786 \\
4.390 & 0.018 & 0.168 & 0.560 & 0.716 \\
5.488 & 0.013 & 0.125 & 0.476 & 0.653 \\
\hline \hline
\end{tabular}

TABLE I. Values for the ratio between the salt concentration in the system, $n_{S}$, and in the reservoir, $n_{S}^{\prime}$, for several Laponite concentrations. These are obtained from semi-grand canonical non-linear PB calculations, at the aspect ratio that minimizes the grand potential. 


\section{FIGURES}

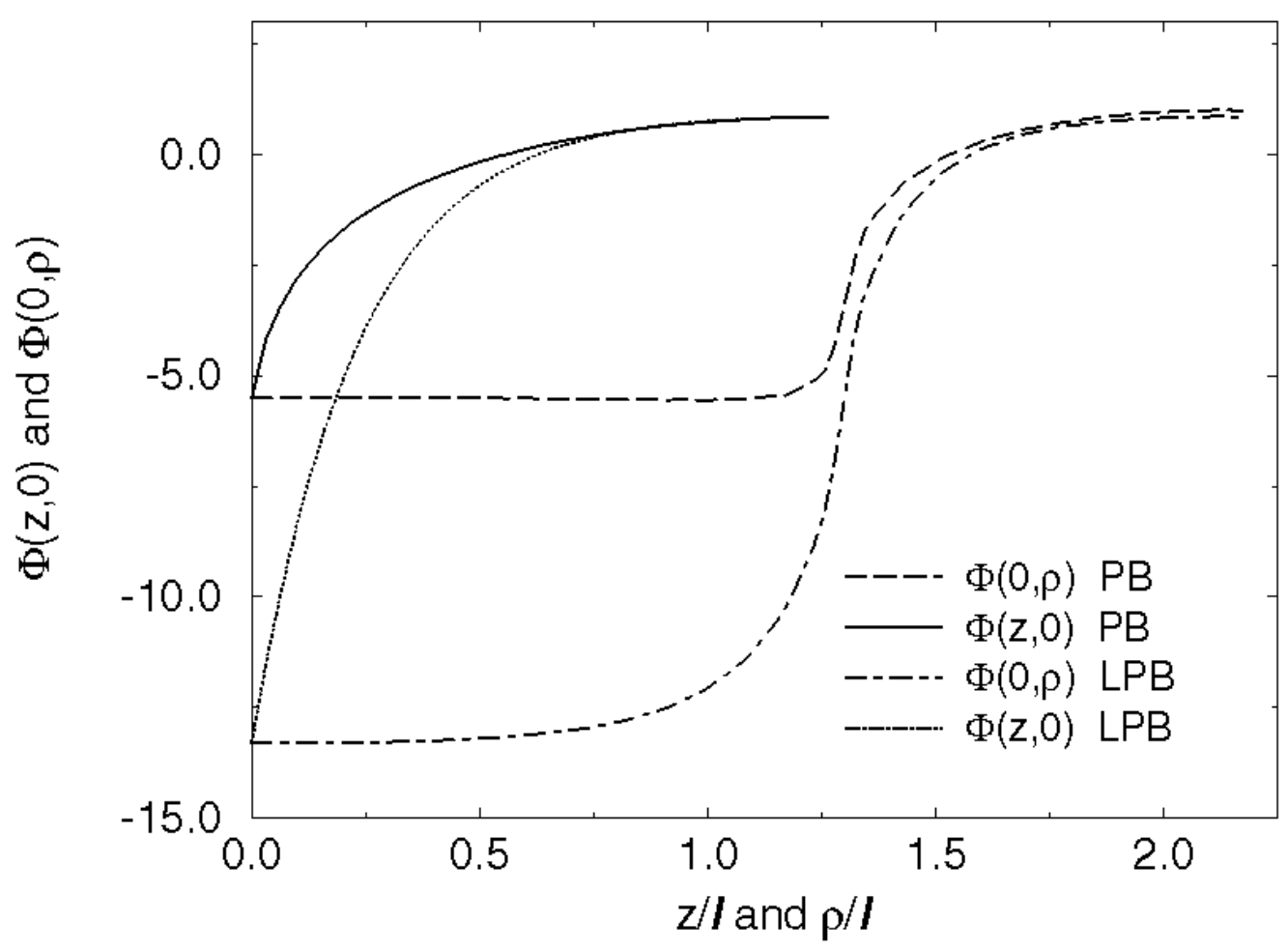

FIG. 1. Dimensionless electrostatic potential $\Phi(z, \rho) \equiv \beta e \varphi(z, \rho)$ at $z=0$ versus $\rho / \ell$, and at $\rho=0$ versus $z / \ell$, with $\ell^{-2}=4 \pi \beta e^{2}\left(2 n_{S}\right) / \epsilon_{0} \epsilon_{r}$. The profiles were obtained in non-linear PB, with the calculations performed in the canonical ensemble, and in LPB (see [8] for details). The results in this figure are for an aqueous solution $\left(\epsilon_{r}=78\right)$ of clay discs of radius $r_{0}=125 \AA$ and surface charge $Z=700$, at a temperature $T=300 \mathrm{~K}$. The concentration of clay is $n=5 \times 10^{-5} \mathrm{M}$ and that of added monovalent salt $n_{S}=10^{-3} \mathrm{M}$. The aspect ratio is $h / r_{0}=0.971$, the value that minimizes the Helmholtz free energy (see Fig 2). 
a)

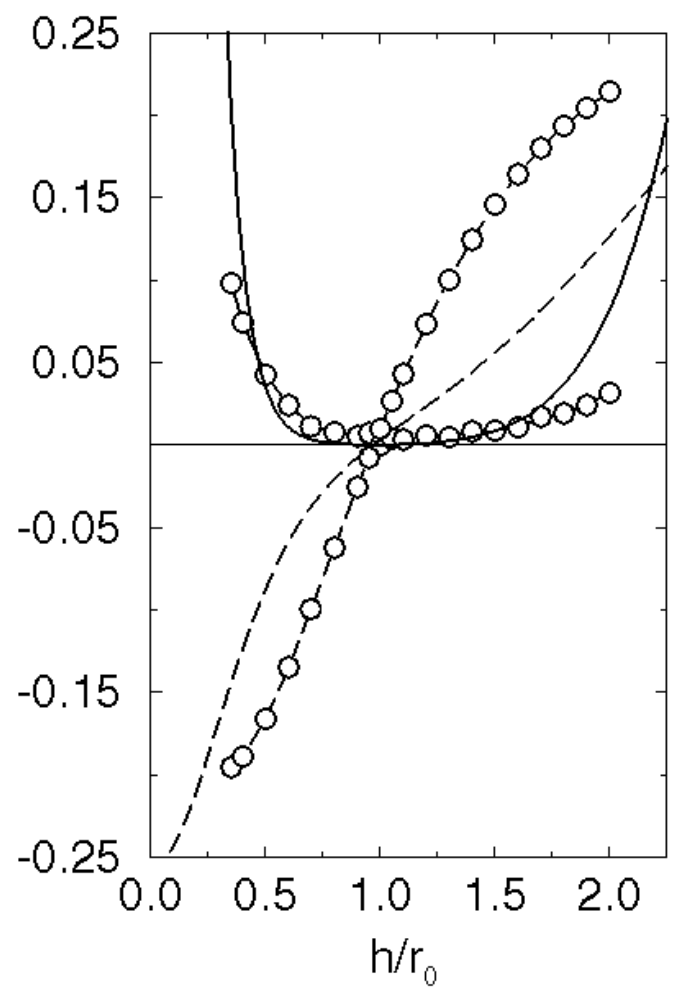

b)

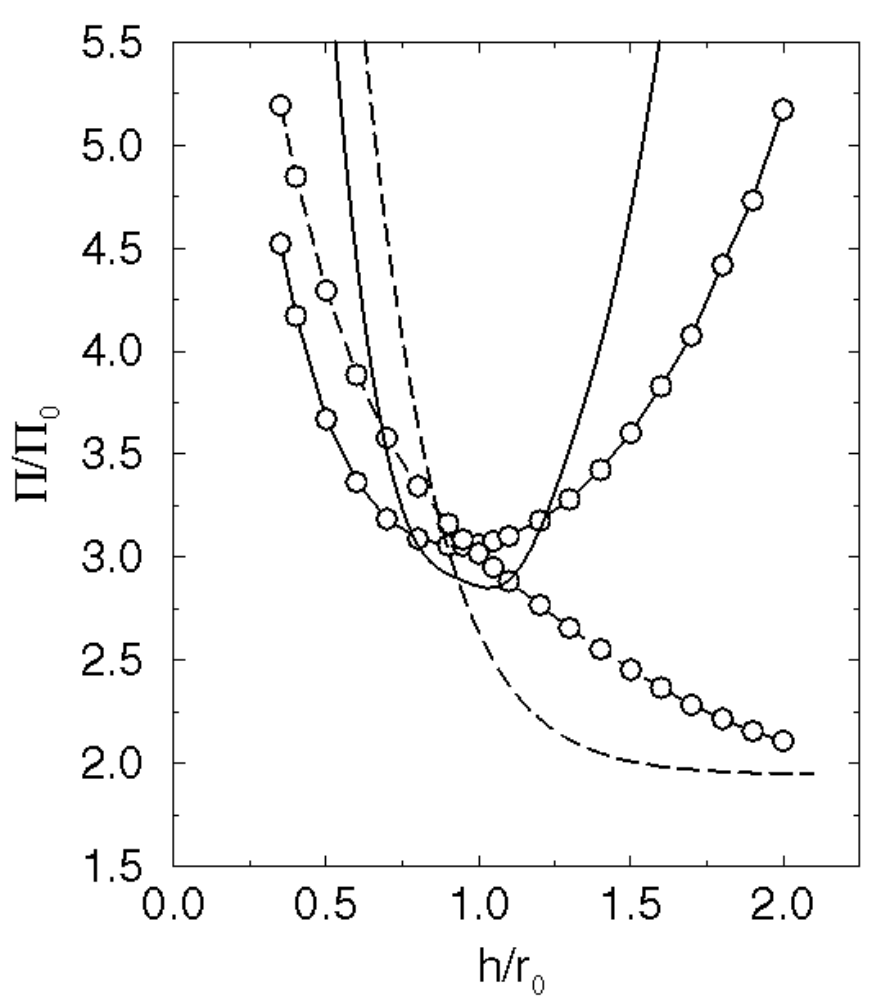

FIG. 2. a) Dependence of the dimensionless Helmholtz free energy $\beta\left(F-F_{0}\right) / Z$ and the dimensionless quadrupole moment $Q / Q^{d i s c}$, with $Q^{d i s c}=Z e r_{0}^{2} / 4$ and $Q=Q_{z z}=-2 Q_{x x}=-2 Q_{y y}$, on the aspect ratio $h / r_{0}$ of the Wigner-Seitz cylindrical cell. The solid lines represent $\beta\left(F-F_{0}\right) / Z$ and the dashed lines are for $Q / Q^{d i s c}$. b) Dependence of the disjoining $\Pi_{d}$ and osmotic $\Pi$ pressure on $h / r_{0}$. The dashed lines correspond to $\Pi_{d}$ and the solid lines to $\Pi$, with $\Pi_{0}=k_{B} T\left(2 n_{S}\right)$. In both plots the curves with $\bigcirc$ were obtained from PB calculations whereas those with no symbols represent LPB results. At the optimum $h / r_{0}$, which minimizes $F, Q$ vanishes and $\Pi=\Pi_{d}$ holds. The optimum value is consistently found to be $h / r_{0}=0.971$, and $F_{0}$ is the value of $F$ at this aspect ratio. The canonical ensemble calculations correspond to the same conditions as in Fig 1 . The grand potential calculated in the semi-grand-canonical ensemble at a reservoir salt concentration $n_{S}^{\prime}=2.54257 \times 10^{-3} \mathrm{M}$ exhibits a minimum at the same value of $h / r_{0}$, where $Q$ vanishes and $\Pi=\Pi_{d}=2530$ Pa from the calculations in both ensembles. 


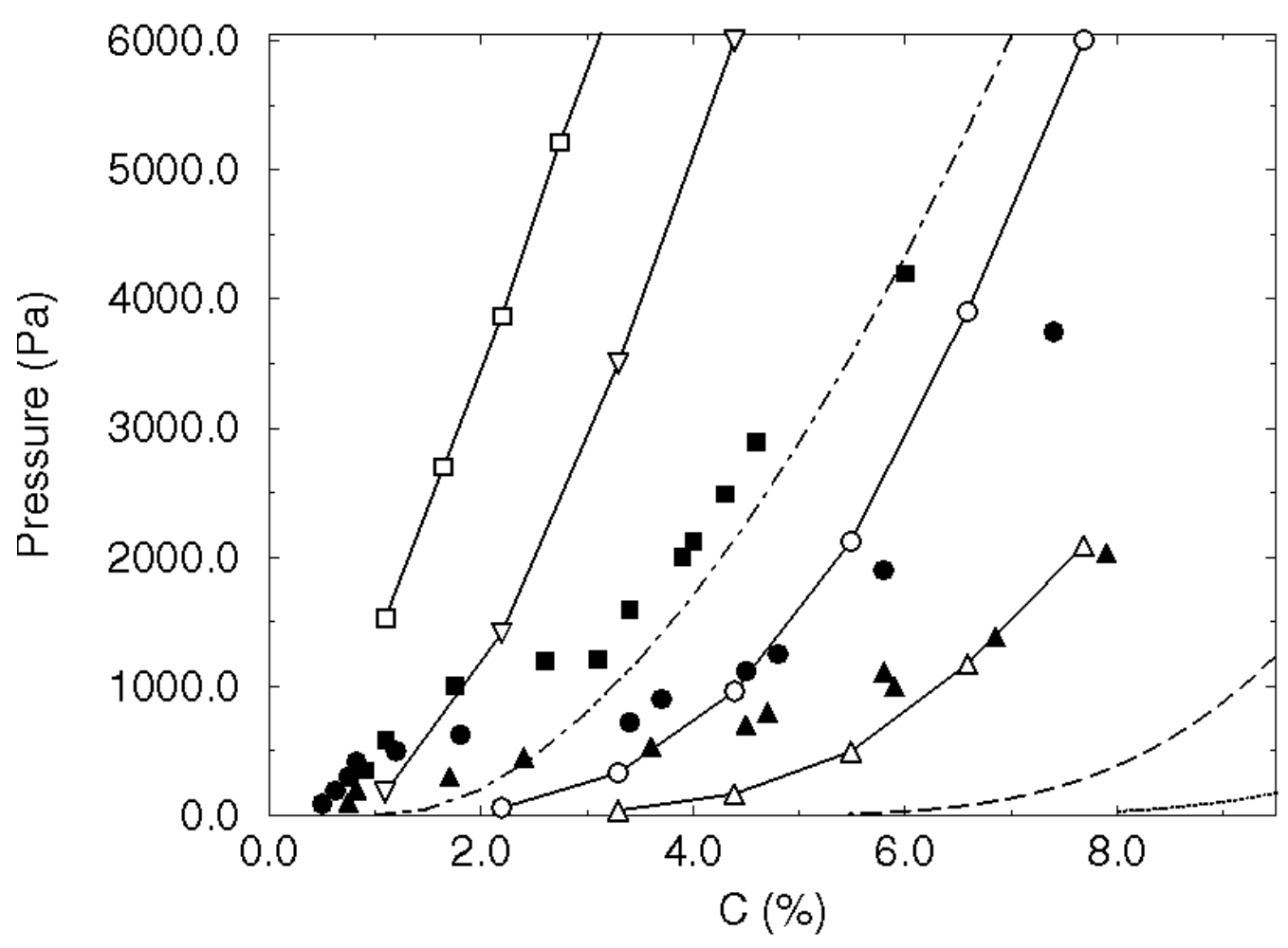

FIG. 3. Equations of state of Laponite suspensions for different reservoir salt concentration $n_{S}^{\prime}$. The filled symbols represent experimental results obtained by Mourchid et al. [2]. The solid lines with open symbols represent our semi-grand canonical PB results obtained at the same values of $n_{S}^{\prime}$. Each of these points corresponds to the osmotic pressure at the minimum of the grand potential with respect to $h / r_{0}$, at fixed $n_{S}^{\prime}$. The values of $n_{S}^{\prime}$ are $10^{-4} \mathrm{M}(\square), 10^{-3} \mathrm{M}(\nabla), 5 \times 10^{-3} \mathrm{M}(\bigcirc)$, and $10^{-2} \mathrm{M}(\triangle)$. The dash-dotted, the dashed and dotted lines are the equations of state obtained from the one-dimensional PB calculation for a stack of infinite planar charged layers in the presence of salt, with the same reservoir concentration $n_{S}^{\prime}$, respectively. The computed results are for an aqueous solution $\left(\epsilon_{r}=78\right)$ of Laponite at $T=300 \mathrm{~K}$. The radius of the clay discs is $r_{0}=150 \AA$ and the surface charge $Z=1000$. 